\title{
ARTICLE OPEN Monolayer WSe2 induced giant enhancement in the spin Hall efficiency of Tantalum
}

\author{
Punyashloka Debashis (D) ${ }^{1,2}$, Terry Y. T. Hung ${ }^{1,2}$ and Zhihong Chen $\mathbb{D}^{1 凶}$
}

Spin Orbit Torque Magnetic RAM (SOT-MRAM) is emerging as a promising memory technology owing to its high endurance, reliability and speed. A critical factor for its success is the development of materials that exhibit efficient conversion of charge current to spin current, characterized by their spin Hall efficiency. In this work, it is experimentally demonstrated that the spin Hall efficiency of the industrially relevant ultra-thin Ta can be enhanced by more than $25 \times$ when a monolayer (ML) WSe $\mathrm{C}_{2}$ is inserted as an underlayer. The enhancement is attributed to spin absorption at the $\mathrm{Ta} / \mathrm{WSe}_{2}$ interface, suggested by harmonic Hall measurements. The presented hybrid spin Hall stack with a $2 \mathrm{D} \mathrm{WSe_{2 }}$ underlayer has a total body thickness of less than $2 \mathrm{~nm}$ and exhibits greatly enhanced spin Hall efficiency, which makes this hybrid a promising candidate for energy efficient SOT-MRAM.

npj 2D Materials and Applications (2020)4:18; https://doi.org/10.1038/s41699-020-0153-z

\section{INTRODUCTION}

SOT-MRAM is a promising alternative to Spin Transfer Torque MRAM, since the novel SOT physics allows for the separation of the read and write current path, thereby improving cell endurance and reliability, and reducing the incubation delay ${ }^{1-3}$. Building energy efficient SOT-MRAM requires innovations in fabricating materials with large spin Hall efficiency. Most work in this regard focuses on engineering the spin-orbit coupling (SOC) strength in heavy metal (HM) materials such as $\mathrm{Ta}, \mathrm{W}$, and $\mathrm{Pt}^{4-6}$. In ultra-thin $\mathrm{HM}$ films, where the thickness becomes comparable to the spin diffusion length of the material, the effective spin Hall angle $\left(\theta_{\mathrm{SHE}}\right)$ is often reduced from the bulk value, due to spin back diffusion ${ }^{7,8}$. However, there could be a different path to improve the spin Hall efficiency, by creating a spin-sink to absorb the unwanted spins and enhance spin accumulation at the interface of the ferromagnet (FM) and spin Hall layer.

In this work, in contrast to the conventional HM films, we present a hybrid spin Hall stack consisting of an ultra-thin Ta layer and a ML WSe $e_{2}$ that exhibits highly efficient spin generation. The $M L \mathrm{WSe}_{2}$ acts as a sink for the spins accumulated at the bottom surface of $\mathrm{Ta}$, thus preventing back diffusion of unwanted spins. Materials with large SOC are known to be effective spin sinks ${ }^{9}$. In fact, transition metal dichalcogenides (TMDs) have been shown to act as good spin sinks in graphene spin valves ${ }^{10,11}$. WSe $\mathrm{W}_{2}$ has been theoretically predicted to have a large SOC among the TMDs studied in literature ${ }^{12-14}$. Therefore, $\mathrm{WSe}_{2}$ is expected to be a good choice for spin sink layer and is experimentally studied in this work. Utilizing this spin sink effect, we demonstrate large spin Hall efficiency in ultra-thin Ta films with thickness of $1 \mathrm{~nm}$. ML WSe is shown to be an excellent spin sink, owing to its large SOC ${ }^{13}$ and large resistivity compared to Ta contributing to negligible current shunting. The desirable combination of large spin Hall efficiency and ultra-thin Ta is presented in terms of a suitable figure of merit for energy efficient SOT switching of magnetization and compared to other reports in literature $4,15,16$.

\section{RESULTS}

Stack deposition and device fabrication

The film stack (starting from the topmost layer) $\mathrm{Ta}(1) / \mathrm{MgO}(1) /$ $\mathrm{CO}_{60} \mathrm{Fe}_{20} \mathrm{~B}_{20}(1) / \mathrm{Ta}\left(\mathrm{t}_{\mathrm{Ta}}\right)$, is prepared by magnetron sputtering for all samples as shown in Fig. 1c. We investigate two types of control samples with $t_{\mathrm{Ta}}=3.5 \mathrm{~nm}$ (stack A) and $t_{\mathrm{Ta}}=1 \mathrm{~nm}$ (stack B), deposited directly on thermally grown $\mathrm{SiO}_{2}$ substrates without any underlayer (Fig. 1a). The test sample (stack C) consists of the film stack with $t_{\mathrm{Ta}}=1 \mathrm{~nm}$ deposited on a $\mathrm{WSe}_{2}$ underlayer, as shown in Fig. 1b. All numbers in brackets above are in nanometer $(\mathrm{nm})$. For the test sample, stack $C$, we start with the transfer of chemical vapor deposition (CVD) grown flakes of $\mathrm{ML} \mathrm{WSe}_{2}$ onto $\mathrm{SiO}_{2}$ substrates, followed by sputtering of the rest of the stack. Raman spectra of the $\mathrm{WSe}_{2}$ film are taken before and after the sputtering deposition, as shown in Fig. $1 \mathrm{~d}$. The $\mathrm{ML} W \mathrm{WS}_{2}$ peaks are present post sputtering, however with a reduced relative peak magnitude, indicating some physical damage to $\mathrm{WSe}_{2}$. However, this is not of great concern, as the function of the $\mathrm{ML} \mathrm{WSe} \mathrm{S}_{2}$ underlayer is not to facilitate lateral current transport, but rather to absorb spins flowing in the vertical direction (from Ta to $\mathrm{ML} W \mathrm{WS}_{2}$ ). In addition, we independently characterize the channel resistance of exfoliated $\mathrm{ML} \mathrm{WSe_{2 }}$ films. Figure 1e shows transfer characteristics at a drain bias of $V_{\mathrm{ds}}=1 \mathrm{~V}$. It is obvious that the total channel resistance of the $\mathrm{ML} \mathrm{WSe}_{2}$ is $>1.5 \mathrm{G} \Omega$ without applied gate voltage, which is orders of magnitude larger than that of Ta. Hence, we conclude that in our hybrid spin Hall stack the current flows in the Ta layer. Hall bar devices are then fabricated for stacks $A, B$, and $C$ by e-beam lithography followed by dry etching using Ar plasma. Figure $1 \mathrm{f}$ shows the optical microscope image of one representative device.

\section{Extracting the value of spin Hall efficiency}

The deposited stacks show perpendicular magnetic anisotropy (PMA) as revealed by anomalous Hall effect (AHE) measurements. Figure $2 a$ shows the measurement configuration. Figure $2 b$ shows the obtained hysteresis loops of the AHE resistance $\left(R_{\omega}\right)$ as a function of Z-directed external magnetic field. From these curves, the value of remanent resistance $\left(R_{\mathrm{A}}\right)$ for all three stacks are

${ }^{1}$ School of Electrical and Computer Engineering and Birck Nanotechnology Center Purdue University, West Lafayette, IN 47907, USA. ${ }^{2}$ These authors contributed equally: Punyashloka Debashis, Terry Y. T. Hung. ${ }^{凶}$ email: zhchen@purdue.edu 
(a)

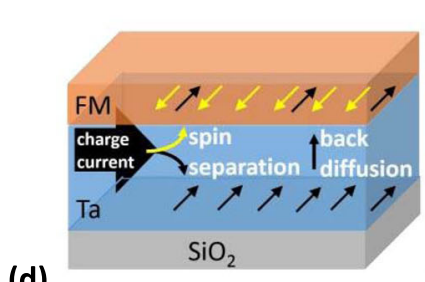

(d)

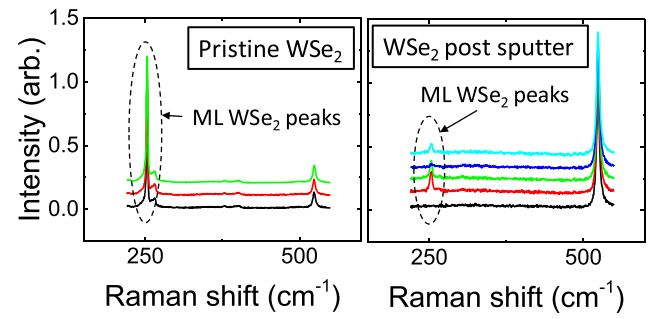

(b) Hybrid SOT layer

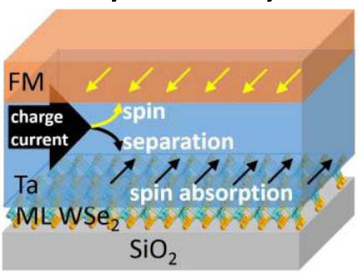

(e) (c) Stacks investigated:

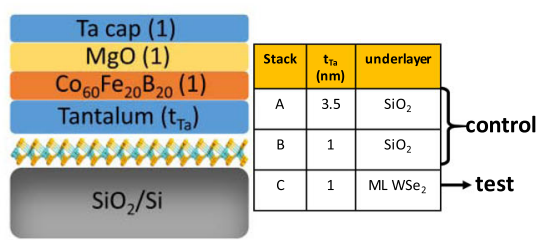

(f)

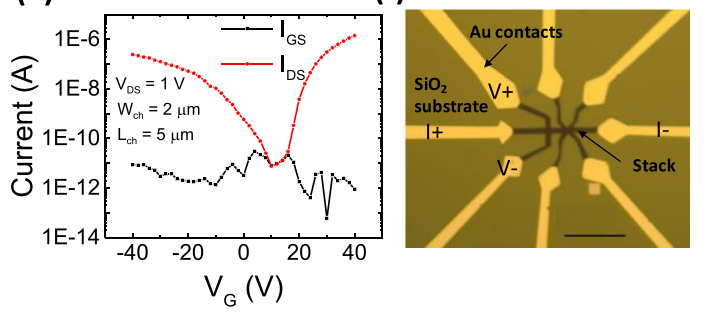

Fig. 1 Effect of underlayer on charge to spin conversion. a Spins accumulated at the $\mathrm{Ta} / \mathrm{SiO}_{2}$ interface result in a vertical concentration gradient of "black" spins, which leads to back diffusion. If the thickness of the Ta layer is comparable to the spin diffusion length, the back diffused spins can be significant and nullify the effect of spin accumulated at the FM interface. $\mathbf{b}$ By inserting a WSe ${ }_{2}$ underlayer, the spins accumulated at the $\mathrm{Ta} / \mathrm{WSe}_{2}$ interface are absorbed by the WSe 2 layer, thus preventing the formation of the vertical spin concentration gradient. Hence, "yellow" spins accumulated on the FM are not nullified, resulting in a better charge to spin conversion efficiency. c The stacks investigated in this work. $\mathbf{d}$ Raman spectra of $\mathrm{MLWSe}_{2}$ before and after magnetic sputter deposition. The peaks corresponding to ML WSe $\mathrm{C}_{2}$ are still preserved after the sputtering process with a reduced relative magnitude, indicative of partial damage to the $M L$ WSe $\mathrm{e}_{2}$ layer. e Transfer characteristics of $\mathrm{ML} \mathrm{WSe}$. The width of the channel is $2 \mathrm{~m}$. The channel resistance is much larger than Ta resistance. $f$ Optical microscope image of a fabricated device with the current and voltage leads marked. The width of the current electrode in this device is also $2 \mu \mathrm{m}$. Length of the scale bar in the image is $20 \mu \mathrm{m}$.
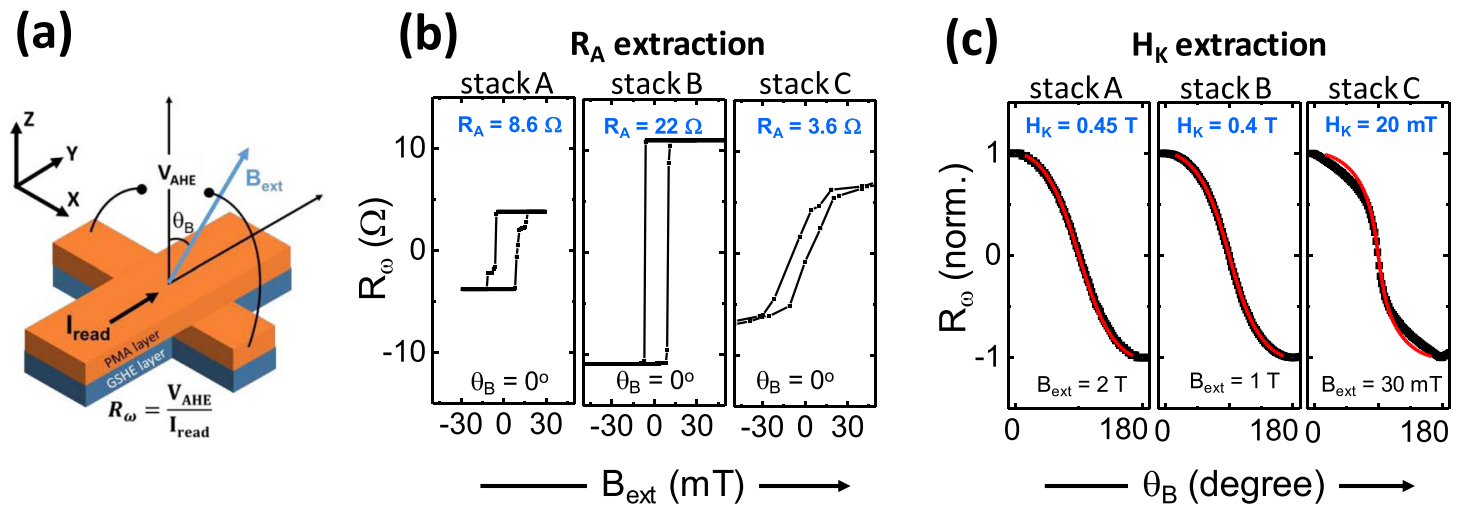

Fig. 2 First harmonic Hall measurements to obtain $\boldsymbol{R}_{\mathrm{A}}$ and $\boldsymbol{H}_{\mathrm{K}}$. a Measurement schematic to obtain $R_{\mathrm{A}}$ and $H_{\mathrm{K}}$. For $R_{\mathrm{A}}$ extraction, the external field $\left(B_{\text {ext }}\right)$ direction is fixed along the $Z$-axis $\left(\theta_{\mathrm{B}}=0^{\circ}\right)$ and its magnitude is swept. For $H_{\mathrm{K}}$ extraction, the magnitude of $B_{\text {ext }}$ is fixed, while its direction is rotated in the $Z-Y$ plane. $R_{\omega}$ is obtained by dividing the first harmonic in phase component of the anomalous Hall voltage by the $\mathrm{R}$. M.S of the AC current excitation. b Measured curves from stacks A, B and C for $R_{\mathrm{A}}$ extraction. Obtained $R_{\mathrm{A}}$ from the remanent values of $R_{\omega}$ at $B_{\text {ext }}=0$ are mentioned in blue text. c Curves obtained from the rotation experiment. The fits to the measured curves give the value of $H_{\mathrm{K}}$ (which are mentioned in blue text in each figure inset).

obtained. The $R_{\mathrm{A}}$ value for device $\mathrm{B}$ is larger than that of $\mathrm{A}$ because a larger fraction of the read current $\left(I_{\text {read }}\right)$ flows through the CoFeB FM layer, owing to the thinner Ta layer. The saturation value of $R_{\omega}$ for device $C$ (at much larger B field, not shown here) is very close to that of device $B$, consistent with the fact that the additional $\mathrm{WSe}_{2}$ layer results in negligible current shunting. However, its $R_{\mathrm{A}}$ value is smaller, possibly because of the FM film breaking into multiple magnetic domains at zero field. This PMA quality can be improved by engineering the deposition conditions as have been shown in literature for other 2-D material underlayers ${ }^{17-19}$. However, in this work, the overall lower quality of PMA in case of stack $C$ compared to the other two stacks is captured by the smaller $R_{\mathrm{A}}$ and a lower $H_{\mathrm{K}}$ (shown later), which are then used to calculate the spin Hall efficiencies in the later sections. This ensures that the calculated spin Hall efficiency is not artificially larger due to a weaker magnetic layer.

Next, the effective perpendicular anisotropy field $\left(H_{\mathrm{K}}\right)$ is obtained by measuring $R_{\omega}$ as the external field is held at a constant magnitude (shown as legends in Fig. 2c) and rotated in the $Y-Z$ plane. The obtained curves are fitted according to the method shown in ref. ${ }^{20}$ to extract $H_{\mathrm{K}}$ values. This method is robust against multi-domain issues as the large external magnetic field ensures coherent rotation of magnetization as a macrospin ${ }^{20}$. The obtained curves along with the fits are shown in Fig. 2c. The obtained $H_{\mathrm{K}}$ values are shown in the figure insets. The possible fitting errors in the extraction of $H_{\mathrm{K}}$ values and their impact on subsequent extraction of spin Hall efficiency are minimal, as shown in Supplementary Fig. 1. 


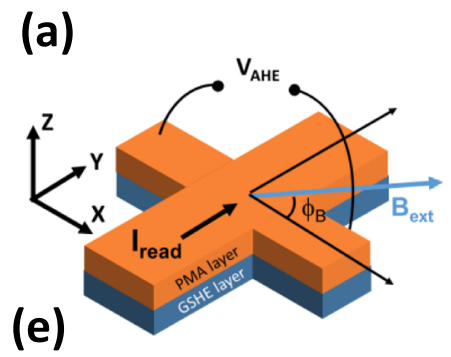

\begin{tabular}{|c|c|c|c|c|}
\hline Stack & $\begin{array}{c}\mathrm{R}_{\mathrm{A}} \\
(\Omega)\end{array}$ & $\begin{array}{c}\mathrm{H}_{\mathrm{K}} \\
(\mathrm{mT})\end{array}$ & $\begin{array}{c}\mathrm{h}_{\mathrm{L}} \\
(\mathrm{Oe})\end{array}$ & $\begin{array}{c}\mathrm{h}_{\mathrm{T}} \\
(\mathrm{Oe})\end{array}$ \\
\hline $\mathrm{A}$ & 8.6 & 450 & 2.6 & 2 \\
\hline B & 22 & 300 & 0.5 & 0.5 \\
\hline $\mathrm{C}$ & 3.6 & 20 & 12.9 & 9.8 \\
\hline
\end{tabular}

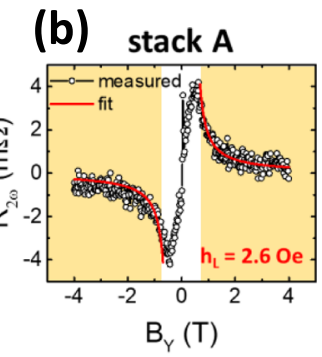

stack A

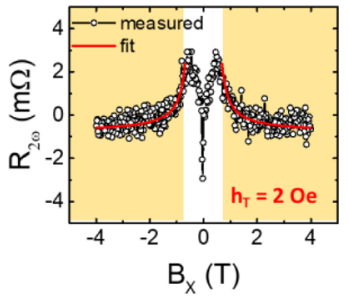

(c) stack B

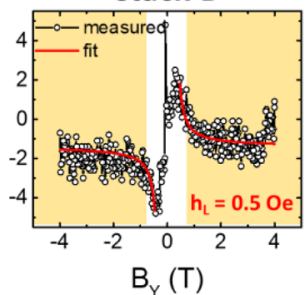

stack B

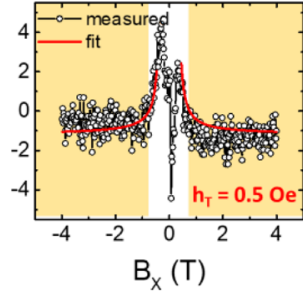

(d)

stack C

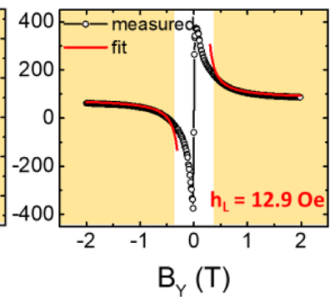

stack C

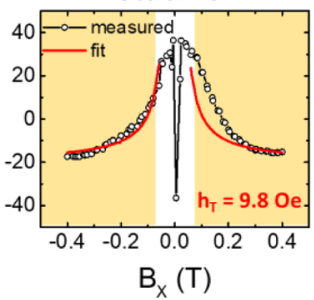

Fig. 3 Second harmonic Hall measurements to obtain $\boldsymbol{h}_{\mathrm{L}}$ and $\boldsymbol{h}_{\mathrm{T}}$. a Measurement schematic to obtain the SOT effective fields. The magnitude of the external magnetic field $\left(B_{\text {ext }}\right)$ is swept while its direction is fixed in the $X-Y$ plane of the film stack, either parallel $\left(\phi_{\mathrm{B}}=90^{\circ}\right)$ or perpendicular $\left(\phi_{\mathrm{B}}=0^{\circ}\right)$ to the current direction. $R_{2 \omega}$ is obtained by dividing the second harmonic quadrature component of the anomalous Hall voltage by the R.M.S of the AC current excitation. Obtained second harmonic response of the devices made from $\mathbf{b}$ stack A, c stack B, and d stack $C$. The top panel shows measurement results for the $\phi_{\mathrm{B}}=90^{\circ}$ configuration and the bottom panel shows that for the $\phi_{\mathrm{B}}=0^{\circ}$ configuration. The longitudinal $\left(h_{\mathrm{L}}\right)$ and transverse $\left(h_{\mathrm{T}}\right)$ effective fields are obtained by fitting the high field regions (highlighted in yellow) of the measured curve (black symbols) with the expression given in Eq. 1 (plotted in red line). The required values of $R_{\mathrm{A}}$ and $H_{\mathrm{K}}$ are obtained previously as shown in Fig. 2. Note here that for the longitudinal configuration, the $R_{2 \omega}$ curve is anti-symmetric while for the transverse configuration it is symmetric, as expected from the sign change of SOT effective fields with the change in magnetization direction. All extracted parameters are listed in the table shown in $\mathbf{e}$.

SOT produces anti-damping and field like torques ${ }^{21}$ on the magnetization that can be characterized as effective fields in the longitudinal $\left(h_{\mathrm{L}}\right)$ and transverse $\left(h_{\mathrm{T}}\right)$ direction, respectively ${ }^{20,22}$. We obtain the values of $h_{\mathrm{L}}$ and $h_{\mathrm{T}}$ from the second harmonic component of the $\mathrm{AHE}$ resistance $\left(R_{2 \omega}\right)$. The measurement configuration is shown in Fig. 3a. Here, the external field is applied in the plane of the film stack, with a direction that is either parallel or perpendicular to the applied current direction for extracting $h_{\mathrm{L}}$ and $h_{\mathrm{T}}$, respectively. In this measurement configuration, $R_{2 \omega}$ is given by:

$$
R_{2 \omega}=-\frac{1}{2} \frac{R_{A} h_{L}}{\left(\left|B_{Y}\right|-H_{K}\right)} ; R_{2 \omega}=-\frac{1}{2} \frac{R_{A} h_{T}}{\left(\left|B_{X}\right|-H_{K}\right)}
$$

where the first and the second expressions correspond to cases when $B_{\text {ext }}$ is parallel and perpendicular to the applied current direction, respectively. In these expressions, the previously obtained values of $R_{\mathrm{A}}$ and $H_{\mathrm{K}}$ are applied leaving $h_{\mathrm{L}}$ or $h_{\mathrm{T}}$ as the only variable in the equation, which can then be obtained by fitting the obtained $R_{2 \omega}$ curve, as shown in Fig. $3 \mathrm{~b}-\mathrm{d}$. This method is also robust against multi-domain issues as the $h_{\mathrm{L}}, h_{\mathrm{T}}$ are obtained by fitting to the large field regions of the $R_{2 \omega}$ curve, where the magnetization is forced to behave as macrospin by the external field. The extracted $h_{\mathrm{L}}$ and $h_{\mathrm{T}}$ values are shown in the respective figure insets and table in Fig. $3 e$. The possible fitting errors in the extraction $h_{\mathrm{L}}$ and $h_{\mathrm{T}}$ values are minimal, as shown in Supplementary Fig. 2.

The $h_{\mathrm{L}}$ and $h_{\mathrm{T}}$ values, normalized by the applied current density $\left(J_{\text {acc }}\right)$ through the Ta layer are indicative of the spin Hall efficiency ${ }^{23}$. For the devices used in our experiments, the width of the current lead is $2 \mu \mathrm{m}$. Together with the film thicknesses and resistivities of Ta and $\mathrm{CoFeB}, J_{\mathrm{ac}}$ is readily calculated. For simplicity, we have assumed that the resistivities of the Ta and the CoFeB layer are the same (which is true for our films grown on $\mathrm{Si} / \mathrm{SiO}_{2}$ ). If a higher resistivity for the Tantalum layer compared to the CoFeB layer is considered, then larger spin Hall efficiency values will be extracted (as the $J_{\text {ac }}$ through the SOT layer will be smaller, hence $h_{\mathrm{L}} / J_{\text {ac }}$ will be larger). Hence, our extracted values for the spin Hall efficiencies are conservative estimates. The obtained $h_{\llcorner} / J_{\text {ac }}$ values for the three stacks are shown in Fig. 4a. For stack $A$, the control sample with $3.5 \mathrm{~nm}$ Ta without the underlayer, our extracted $h_{\mathrm{L}} / J_{\mathrm{ac}}$ value is consistent with previously reported in literature ${ }^{4}$. For stack $\mathrm{B}$, the control sample with 1-nm Ta without the underlayer, the $h_{\mathrm{L}} /$ $J_{\mathrm{ac}}$ value reduces as $t_{\mathrm{Ta}}=1 \mathrm{~nm}$ is smaller than the spin diffusion length in Ta, as will be explained in the next section. The $h_{\mathrm{L}} / J_{\mathrm{ac}}$ value for stack $C$, our test sample with $1 \mathrm{~nm}$ Ta and the ML WSe underlayer, has improved significantly (by $26.5 \times$ ), owing to the suppression of the spin back diffusion since a large fraction of these unwanted spins are absorbed at the $\mathrm{Ta} / \mathrm{WSe}_{2}$ interface. Also shown in the same plot are the ratio of the longitudinal effective field to the transverse effective field $\left(\mathrm{h}_{T} / \mathrm{h}_{\mathrm{L}}\right)$. As can be seen, this ratio does not change significantly among the three stacks, which suggests the same SOT mechanism occurring in all three stacks.

The spin sink effectiveness can be directly obtained through spin pumping experiments by measuring the Gilbert damping constant of the CoFeB/Ta stack as a function of the Ta layer thickness, with and without the WSe $\mathrm{W}_{2}$ underlayer as done for other material systems ${ }^{9}$. However, the goal in our paper is to focus on the application of this effect to improve the power efficiency of an SOT based device. Therefore, we have measured and quantified the spin Hall efficiency directly and then corroborated it with the spin sink effect.

\section{DISCUSSION}

The spin Hall angle $\left(\theta_{\mathrm{SHE}}\right)$ is the most commonly used metric to evaluate spin Hall efficiency. $\theta_{\text {SHE }}$ can be calculated from the $\left(h_{\mathrm{L}} /\right.$ $\left.J_{\mathrm{ac}}\right)$ values through the following expression ${ }^{20}$ :

$\theta_{\mathrm{SHE}}=\frac{2 e}{\hbar} \frac{h_{\mathrm{L}}}{J_{\mathrm{ac}}} M_{\mathrm{S}} t_{\mathrm{FM}}$

where $e$ is the charge of an electron, $\hbar$ is the reduced Planck constant $(h / 2 \pi) . t_{\mathrm{FM}}$ is the thickness of the FM layer and $M_{\mathrm{S}}$ is its saturation magnetization. In our stacks, $t_{\mathrm{FM}}=t_{\mathrm{COFeB}}=1 \mathrm{~nm}$. $M_{\mathrm{S}}$ is measured using Superconducting Quantum Interference Device (SQUID) 
(a)

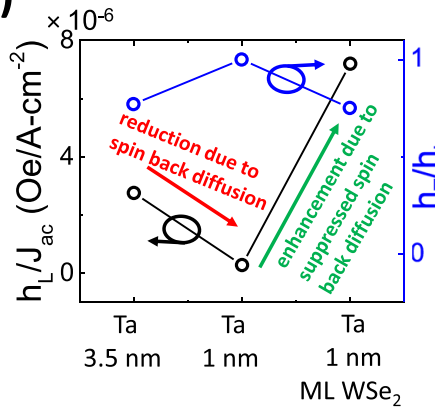

(b)

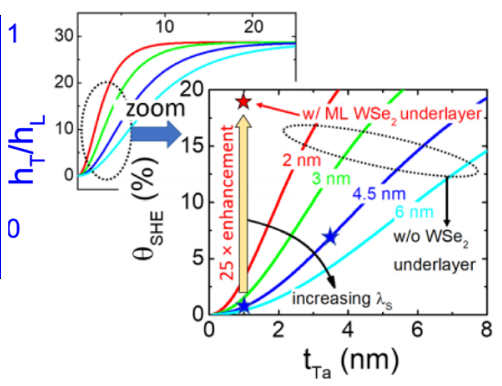

(c)

\begin{tabular}{c|c|c|c|c|}
\hline$I_{C}^{2} R=\left(\frac{2 e}{\hbar}\right)^{2}\left(\frac{E_{B}^{2}}{W L}\right) /\left(\frac{\theta_{S H E}^{2}}{\rho \times t_{S H E}}\right)$ \\
\hline $\begin{array}{c}\text { GSHE } \\
\text { stack }\end{array}$ & $\begin{array}{c}\theta_{\text {SHE }} \\
(\%)\end{array}$ & $\begin{array}{c}\mathrm{t}_{\text {SHE }} \\
(\mathrm{nm})\end{array}$ & $\begin{array}{c}\rho \\
(\mu \Omega-\mathrm{cm})\end{array}$ & $\begin{array}{c}\frac{\theta_{S H E}^{2}}{\rho \times t_{\text {SHE }}} \\
\left(10^{3} / \mu \Omega-\mathrm{cm}^{2}\right)\end{array}$ \\
\hline$\beta-\mathrm{Ta}^{4}$ & 12 & 4 & 189 & 0.18 \\
\hline$\beta-\mathrm{Ta}^{15}$ & 13 & 7.3 & 185 & 0.12 \\
\hline$\beta-\mathrm{Ta}^{16}$ & 2.5 & 3 & 210 & 0.01 \\
\hline $\begin{array}{c}\left(\mathrm{Ta} / \text { WSe }_{2}\right) \\
\text { This work }\end{array}$ & 18.6 & 1 & 462 & $\mathbf{0 . 7 5}$ \\
\hline
\end{tabular}

Fig. 4 Spin Hall efficiency of the hybrid $\mathbf{W S e} \mathbf{e}_{2} / \mathbf{T a}$ stack compared to standard SOT stacks. a Spin Hall efficiency expressed by ratio of longitudinal effective field to the current density for the devices made from the three different stacks. When Ta thickness is decreased from $3.5 \mathrm{~nm}$ to $1 \mathrm{~nm}$, the spin Hall efficiency decreases to nearly zero due to significant back diffusion of spins from the Ta/SiO ${ }_{2}$ interface. When a $\mathrm{ML} \mathrm{WSe}_{2}$ is inserted, the spin Hall efficiency is greatly improved. The ratio of the longitudinal and transverse effective field is plotted with the right $y$-axis of the graph. We can see that the ratio does not show such huge change, suggesting that the mechanism of SOT is not altered. b The effective spin Hall angle $\left(\theta_{\text {SHE }}\right)$ increases as a function of the Ta layer thickness according to Eq. 3. The experimentally obtained $\theta_{\text {SHE }}$ for stacks $A$ and $B$ (shown with the filled blue symbols) are well fitted with a spin diffusion length of $4.5 \mathrm{~nm}$. With the insertion of ML WSe ${ }_{2}$, the experimentally obtained $\theta_{\text {SHE }}$ (shown with the red star) is closer to the intrinsic bulk limit value. c Comparison of obtained $\theta_{\text {SHE }}$ and Ta thickness of our stacks with that presented in literature. A combination of high $\theta_{\text {SHE }}$ and ultra-thin Ta is desirable for energy efficient SOT switching of magnetization, captured by the figure of merit shown in the last column.

magnetometry with MPMS-3 to be $1031 \mathrm{emu} / \mathrm{cc}$, which is consistent with the quoted value in literature $\mathrm{Co}_{60} \mathrm{Fe}_{20} \mathrm{~B}_{20}{ }^{24}$. The magnetometry data of stacks $B$ and $C$ are shown in Supplementary Fig. 3.

The giant spin Hall effect results in the separation of oppositely polarized spins to the top and bottom surface of Ta. The spins reaching the top surface are absorbed by the FM layer and contribute to the observed SOT. However, the spins reaching the bottom surface of Ta get accumulated and as a result create a concentration gradient in the vertical direction. This leads to back diffusion of the oppositely directed spins into the FM, hence partly nullifying the effect of SOT. This is captured by the following expression for the measured $\theta_{\mathrm{SHE}}{ }^{25}$ :

$\theta_{\mathrm{SHE}}=\theta_{\mathrm{SHE}}^{\text {bulk }} \times\left(1-\operatorname{sech}\left(\frac{t_{\mathrm{Ta}}}{\lambda_{\mathrm{S}}}\right)\right)$

where $\theta_{S H E}^{\text {bulk }}$ is the spin Hall angle in the bulk limit and $\lambda_{S}$ is the spin diffusion length in Ta. $\theta_{\text {SHE }}$ obtained from the above expression is plotted as a function of $t_{\mathrm{Ta}}$ for various $\lambda_{S}$ in Fig. $4 \mathrm{~b}$. From the $\theta_{\mathrm{SHE}}$ values for stacks $A$ and $B$, we extract $\lambda_{S}=4.5 \mathrm{~nm}$ and $\theta_{\mathrm{SHE}}^{\text {bulk }}=28.7 \%$ by fitting the above equation, consistent with previously reported values in literature ${ }^{7}$.

Now, for stack $C$, the spins reaching the bottom surface of Ta are absorbed by the $\mathrm{WSe}_{2}$ underlayer, resulting in suppressed back diffusion. Hence, the experimentally extracted value of $\theta_{\mathrm{SHE}}$ for stack $C$ should be much closer to the $\theta_{S H E}^{\text {bulk value, which is }}$ indeed confirmed in our experiment, as seen from the experimentally obtained data point (red star) for stack $C$ in Fig. $4 \mathrm{~b}$. This indicates that the use of $\mathrm{WSe}_{2}$ underlayer restores the value of spin Hall angle closer to the bulk value even for the case of ultrathin Ta layers. The improvement in spin Hall angle is quantified by $\theta_{\text {SHE }}($ stack C $) / \theta_{\text {SHE }}($ stack B $)=26.5$.

In order to test the structural differences in the Ta layer itself due to the presence of a $\mathrm{WSe}_{2}$ underlayer in stack $\mathrm{C}$ vs. $\mathrm{SiO}_{2}$ in stack $B$, we fabricated 4 probe devices and measured the resistivities. The resistivity of stack $B$ was measured to be $320-$ $\mu \Omega-\mathrm{cm}$ and that of stack $C$ to be $462-\mu \Omega-\mathrm{cm}$. These resistivity values are close to the range of values reported in literature. For example, Zhu et al. ${ }^{26}$ report a resistivity value of $\sim 330-\mu \Omega-\mathrm{cm}$ and $\mathrm{Tao}^{27}$ reports a value of $\sim 400-\mu \Omega-\mathrm{cm}$ for similar stacks. Yu et al. ${ }^{7}$ and $\mathrm{Qu}$ et al. ${ }^{28}$ report values of $400-\mu \Omega-\mathrm{cm}$ and $\sim 530-\mu \Omega-\mathrm{cm}$ respectively for a tantalum layer thickness of $2 \mathrm{~nm}$. Therefore, we believe that the tantalum layer in both of our samples is in the standard $\beta$ phase. However, there could be contribution to the observed enhancement in spin Hall efficiency coming from slightly changed microstructure of the Ta layer, which requires further study beyond the scope of this paper.

Spin Hall angle, resistivity and the thickness of the SOT layer all are important parameters that impact the power efficiency of an SOT based memory device. Therefore, it is incomplete to consider the improvement in one of these parameters in isolation. We considered a figure of merit that captures their convoluted impact on the SOT switching power in the following way:

The power required for SOT switching of a PMA magnet with an energy barrier $\left(E_{\mathrm{B}}\right)$ can be obtained from the expression for the critical switching current $\left(I_{C}\right)^{4}$ :

$P_{\text {switching }}=I_{\mathrm{C}}^{2} R=\left(\frac{2 e}{\hbar}\right)^{2}\left(\frac{E_{\mathrm{B}}^{2}}{W L}\right) /\left(\frac{\theta_{\mathrm{SHE}}^{2}}{\rho \times t_{\mathrm{SHE}}}\right)$

Since $E_{\mathrm{B}}$ and dimensions of the magnet $(W, L)$ are predetermined by the desired retention time and targeted technology node respectively, the denominator, $\left(\frac{\theta_{S \mathrm{SH}}^{2}}{\rho \times t_{\mathrm{SHE}}}\right)$ becomes an important figure of merit. Figure $4 c$ compares $\theta_{\mathrm{SHE}}$ and this figure of merit, where the presented $\mathrm{Ta} / \mathrm{WSe}_{2}$ stack outperforms the conventional giant spin Hall material $\beta$-Ta by a factor $>4$. Our experiments suggest that efficient absorption of spins at the $\mathrm{Ta} /$ $\mathrm{WSe}_{2}$ interface is responsible for this giant enhancement by preventing spin back diffusion.

In summary, we have demonstrated a hybrid SOT stack that shows enhanced spin Hall efficiency compared to the standard $\beta$-Ta. This finding shows a promising integration of $2 \mathrm{D}$ materials in spintronics devices for energy efficient SOT-MRAM applications.

\section{METHODS}

Sample preparation and characterization

Chemical vapor deposition (CVD) grown monolayer $\mathrm{WSe}_{2}$ flakes were transferred to a silicon substrate with $90 \mathrm{~nm}$ thermal $\mathrm{SiO}_{2}$. Raman spectroscopy was performed after this step to confirm the layer number of $\mathrm{WSe}_{2}$ flakes. PVD magnetron sputtering was then used to deposit the stacks $A, B$, and C mentioned in the main text, followed by another Raman spectroscopy step.

\section{Device Fabrication}

The devices were fabricated in a two-step e-beam lithography process. First, the entire stack was etched into a Hall bar shape by e-beam lithography and dry etching using Argon plasma, followed by removal of the HSQ etch mask. Then, the contact pads were formed on the Hall bar by 
another e-beam lithography step followed by e-beam evaporation of $\mathrm{Ti}$ $(20 \mathrm{~nm}) / \mathrm{Au}(100 \mathrm{~nm})$ and liftoff in acetone.

For obtaining the transfer characteristics of the monolayer $\mathrm{WSe}_{2}$ devices as shown in Fig. 1e, only one e-beam lithography step was used to define the contacts on transferred flakes, followed by e-beam evaporation of $\mathrm{Ti}$ $(20 \mathrm{~nm}) / \mathrm{Au}(100 \mathrm{~nm})$ and liftoff in acetone.

\section{Measurement setup}

The transfer characteristics on Fig. 1e were obtained using Agilent semiconductor parameter analyzer with a Lakeshore probe station. The harmonic Hall measurements were performed by using a sinusoidal current from a Keithley 6221 current source and an SRS 850 DSP lock-in amplifier. These measurements were carried out inside a Quantum Design PPMS Dynacool system, with the sample mounted on a rotatable stage that allowed to change the angles $(\theta$ and $\phi)$ of the external magnetic field w.r.t to the film normal and current direction from $0^{\circ}$ to $90^{\circ}$.

\section{DATA AVAILABILITY}

The data that support the findings of this study are available from the corresponding author upon reasonable request.

Received: 24 September 2019; Accepted: 3 June 2020; Published online: 23 June 2020

\section{REFERENCES}

1. Garello, K. et al. SOT-MRAM 300MM integration for low power and ultrafast embedded memories. In 2018 IEEE Symposium on VLSI Circuits 81-82 (IEEE, 2018).

2. Bhatti, S. et al. Spintronics based random access memory: a review. Mater. Today 20, 530-548 (2017).

3. Wang, K. L., Alzate, J. G. \& Khalili Amiri, P. Low-power non-volatile spintronic memory: STT-RAM and beyond. J. Phys. D Appl. Phys. 46, 074003 (2013).

4. Liu, L. et al. Spin-torque switching with the giant spin hall effect of tantalum. Science 336, 555-558 (2012).

5. Pai, C.-F. et al. Spin transfer torque devices utilizing the giant spin Hall effect of tungsten. Appl. Phys. Lett. 101, 122404 (2012).

6. Liu, L., Moriyama, T., Ralph, D. C. \& Buhrman, R. A. Spin-torque ferromagnetic resonance induced by the spin hall effect. Phys. Rev. Lett. 106, 036601 (2011).

7. $\mathrm{Yu}, \mathrm{R}$. et al. Determination of spin Hall angle and spin diffusion length in $\beta$-phase-dominated tantalum. Phys. Rev. Mater. 2, 074406 (2018).

8. Jiao, H. \& Bauer, G. E. W. Spin backflow and ac voltage generation by spin pumping and the inverse spin hall effect. Phys. Rev. Lett. 110, 217602 (2013).

9. Tserkovnyak, Y., Brataas, A., Bauer, G. E. W. \& Halperin, B. I. Nonlocal magnetization dynamics in ferromagnetic heterostructures. Rev. Mod. Phys. 77, 1375-1421 (2005).

10. Yan, W. et al. A two-dimensional spin field-effect switch. Nat. Commun. 7, 13372 (2016).

11. Dankert, A. \& Dash, S. P. Electrical gate control of spin current in van der Waals heterostructures at room temperature. Nat. Commun. 8, 16093 (2017).

12. Xiao, D. et al. Coupled spin and valley physics in monolayers of MoS 2 and other group-VI dichalcogenides. Phys. Rev. Lett. 108, 196802 (2012).

13. Zhu, Z. Y., Cheng, Y. C. \& Schwingenschlögl, U. Giant spin-orbit-induced spin splitting in two-dimensional transition-metal dichalcogenide semiconductors. Phys. Rev. B 84, 153402 (2011).

14. Feng, W. et al. Intrinsic spin Hall effect in monolayers of group-VI dichalcogenides: a first-principles study. Phys. Rev. B Condens. Matter Mater. Phys. 86, 165108 (2012).

15. Allen, G., Manipatruni, S., Nikonov, D. E., Doczy, M. \& Young, I. A. Experimental demonstration of the coexistence of spin Hall and Rashba effects in $\beta$-tantalum/ ferromagnet bilayers. Phys. Rev. B Condens. Matter Mater. Phys. 91, 144412 (2015).

16. Kim, S. I., Kim, D. J., Seo, M. S., Park, B. G. \& Park, S. Y. Dependence of inverse-spin Hall effect and spin-rectified voltage on tantalum thickness in $\mathrm{Ta} / \mathrm{CoFeB}$ bilayer structure. Appl. Phys. Lett. 106, 032409 (2015).

17. Shao, Q. et al. Strong rashba-edelstein effect-induced spin-orbit torques in monolayer transition metal dichalcogenide/ferromagnet bilayers. Nano Lett. 16, 7514-7520 (2016).

18. Yang, $\mathrm{H}$. et al. Anatomy and giant enhancement of the perpendicular magnetic anisotropy of cobalt-graphene heterostructures. Nano Lett. 16, 145-151 (2016).

19. Zhang, W. et al. Ferromagnet/two-dimensional semiconducting transition-metal dichalcogenide interface with perpendicular magnetic anisotropy. ACS Nano 13, 2253-2261 (2019).
20. Fan, Y. et al. Magnetization switching through giant spin-orbit torque in a magnetically doped topological insulator heterostructure. Nat. Mater. 13, 699-704 (2014).

21. Sun, J. Z. Spin-current interaction with a monodomain magnetic body: a model study. Phys. Rev. B 62, 570-578 (2000).

22. Hayashi, M., Kim, J., Yamanouchi, M. \& Ohno, H. Quantitative characterization of the spin-orbit torque using harmonic Hall voltage measurements. Phys. Rev. B Condens. Matter Mater. Phys. 89, 144425 (2014).

23. Garello, K. et al. Symmetry and magnitude of spin-orbit torques in ferromagnetic heterostructures. Nat. Nanotechnol. 8, 587-593 (2013).

24. Shaw, J. M. et al. Perpendicular magnetic anisotropy and easy cone state in $\mathrm{Ta} /$ $\mathrm{CO}_{60} \mathrm{Fe}_{20} \mathrm{~B}_{20} / \mathrm{MgO}$. IEEE Magn. Lett. 6, 1-4 (2015).

25. Liu, L., Lee, O. J., Gudmundsen, T. J., Ralph, D. C. \& Buhrman, R. A. Current-induced switching of perpendicularly magnetized magnetic layers using spin torque from the spin hall effect. Phys. Rev. Lett. 109, 096602 (2012).

26. Zhu, T., Chen, P., Zhang, Q. H., Yu, R. C. \& Liu, B. G. Giant linear anomalous Hall effect in the perpendicular CoFeB thin films. Appl. Phys. Lett. 104, 202404 (2014).

27. Zhu, T. Anomalous Hall effect in perpendicular CoFeB thin films. Chinese Phys $B$ 23, 047504 (2014).

28. Qu, D., Huang, S. Y., Miao, B. F., Huang, S. X. \& Chien, C. L. Self-consistent determination of spin Hall angles in selected $5 \mathrm{~d}$ metals by thermal spin injection. Phys. Rev. B 89, 140407-140408 (2014).

\section{ACKNOWLEDGEMENTS}

We would like to thank Dr. Kerem Camsari, Prof. Joerg Appenzeller, and Prof. Pramey Upadhyaya for fruitful discussions and their feedback on this work. This work was supported by the Center for Probabilistic Spin Logic for Low-Energy Boolean and Non-Boolean Computing (CAPSL), one of the Nanoelectronic Computing Research (nCORE) Centers as task 2759.003 and 2759.004, a Semiconductor Research Corporation (SRC) program sponsored by the NSF through CCF 1739635.

\section{AUTHOR CONTRIBUTIONS}

P.D and T.Y.T.H performed the sample fabrication and characterization under Z.C's supervision. All authors performed data analysis and wrote the manuscript. P.D. and T.Y.T.H. are co-first authors.

\section{COMPETING INTERESTS}

The authors declare no competing interests.

\section{ADDITIONAL INFORMATION}

Supplementary information is available for this paper at https://doi.org/10.1038/ s41699-020-0153-z.

Correspondence and requests for materials should be addressed to Z.C.

Reprints and permission information is available at http://www.nature.com/ reprints

Publisher's note Springer Nature remains neutral with regard to jurisdictional claims in published maps and institutional affiliations.

\begin{abstract}
Open Access This article is licensed under a Creative Commons Attribution 4.0 International License, which permits use, sharing, daptation, distribution and reproduction in any medium or format, as long as you give appropriate credit to the original author(s) and the source, provide a link to the Creative Commons license, and indicate if changes were made. The images or other third party material in this article are included in the article's Creative Commons license, unless indicated otherwise in a credit line to the material. If material is not included in the article's Creative Commons license and your intended use is not permitted by statutory regulation or exceeds the permitted use, you will need to obtain permission directly from the copyright holder. To view a copy of this license, visit http://creativecommons. org/licenses/by/4.0/.
\end{abstract}

(c) The Author(s) 2020 